\title{
28 Research Square \\ SNRPB is a Mediator for Cellular Response to Cisplatin in Non-Small-Cell Lung Cancer
}

Nianli Liu ( $\square$ liunli@xzhmu.edu.cn )

Cancer Institute of Xuzhou Medical University https://orcid.org/0000-0002-0602-6709

Aoxing Chen

Cancer Institute of Xuzhou Medical University

Ning Feng

Cancer Institute of Xuzhou Medical University

Xiaochen Liu

Yanzhou District, the Affiliated Hospital of Jining Medical University

Longzhen Zhang

Center of Clinical Oncology, Affiliated Hospital of Xuzhou Medical University

\section{Research}

Keywords: SNRPB, NSCLC, Cisplatin, Resistance, Biomarker

Posted Date: November 24th, 2020

DOl: https://doi.org/10.21203/rs.3.rs-112901/v1

License: (c) (1) This work is licensed under a Creative Commons Attribution 4.0 International License.

Read Full License 


\section{Abstract}

Background: Our previous study has demonstrated that small nuclear ribonucleoprotein polypeptides B And $\mathrm{B}^{\prime}$ (SNRPB) is highly expressed in non-small-cell lung cancer (NSCLC) and functions as an oncogene. However, whether SNRPB contributes to cisplatin resistance in NSCLC is still unknown. This study aimed to explore how SNRPB regulates the effect of cisplatin in NSCLC.

Methods: In this study, cell counting kit-8 (CCK-8) and flow cytometry assays were performed to examine cell survival, cell cycle and apoptosis in NSCLC cells upon cisplatin treatment. Western blotting assays were used to examine the cell cycle and apoptosis-related protein expression. The effects of SNRPB on cisplatin-mediated tumor inhibition was measured via a xenograft tumor model in nude mouse.

Results: SNRPB negatively regulates cisplatin resistance in NSCLC cells. Knocking out of SNRPB could significantly decrease cisplatin-induced cell growth inhibition, cell cycle arrest and apoptosis in $\mathrm{H} 1299$ cells. However, enforced expression of SNRPB in H460 cells can markedly promote cisplatin-induced cell growth inhibition, cell cycle arrest and apoptosis. Our results also indicate that overexpression of SNRPB enhances the inhibitory effects of cisplatin on $\mathrm{H} 460$ cell-mediated xenograft tumors.

Conclusion: Our results suggest that SNRPB may be a prediction marker for NSCLC patients in response to cisplatin-based chemotherapy.

\section{Introductin}

In the past decades, lung cancer ranks as one of the most common human cancers and is among the leading causes of mortality. It can be divided into two main types: small cell lung cancer (SCLC) and nonsmall-cell lung cancer (NSCLC). NSCLC accounts for $\$ 85 \%$ of lung cancer diseases[1-3], however, for more than half of patients already diagnosed as advanced or metastatic lung cancer when it was found. Multiple methods have been used for the treatment of NSCLC, including surgical treatment, which is the main approach for early-stage lung cancer[4]. Chemotherapy is also an option and an important adjuvant therapy in addition to surgery. Among these, cisplatin (CDDP) is the mainstay treatment for NSCLC with benefits like diminutive tumor size and improving prognosis[5, 6]. However, the efficacy of cisplatin is not adequate due to the highly effective DNA replication and repair system in cancer cells. Besides, the application of cisplatin is limited because of reduced chemosensitivity and toxicity. Therefore, it is particularly important to identify predictive markers for patients with NSCLC in response to cisplatinbased chemotherapy.

SNRPB is a core component of spliceosome and acts as a splicing factor. It plays a critical role in the alternative splicing process of pre-mRNA [7]. Alternative splicing regulation has recently emerged as a significant mechanism in cancer development and progression. It has proved that alternative splicing can promote tumor aggressiveness and drug resistance in African American prostate cancer[8]. Many studies that have reported that alternative splicing mediates cisplatin resistance in cancer cells. For example, a splice variant of BRCA1 lacking exon 11 confers cisplatin resistance in breast and ovarian cancer 
patients [9]. The inhibition of major splicing factors SRPK1 can sensitize ovarian cancer cells to cisplatin treatment [10].

As a splicing factor, whether SNRPB regulates cisplatin resistance remains unknown. Several recent studies reported that SNRPB is elevated and acts as an oncogene in multiple cancers, such as NSCLC, liver cancer, cervical cancer, glioblastoma [11-14]. In these cancers, SNRPB exhibits its oncogenic function by alternative splicing the pre-mRNA of downstream targets. In this study, we sought to examine whether SNRPB modulates NSCLC cells in response to cisplatin treatment.

\section{Materials And Methods}

\section{Cell cultures}

All cell lines used in this study were cultured as previously described [12]. In brief, cells were cultured in DMEM medium with $10 \% \mathrm{FBS}$ at $37^{\circ} \mathrm{C}$ and $5 \% \mathrm{CO} 2$.

\section{Antibodies and reagents}

SNRPB mouse monoclonal antibody (Y12) was obtained from Invitrogen. Actin, CDC5L, CCNB1, CDK4 and GAPDH antibodies were from Abclonal. PARP, $\mathrm{y}-\mathrm{H} 2 \mathrm{AX}$, pERK and ERK primary antibodies were purchaesd from Cell Signaling Technology. Cisplatin was purchased from MedChemExpress.

\section{CCK-8 assay}

$\mathrm{H} 1299$ and $\mathrm{H} 460$ cells were seeded into 96 -well plates at the density of $2 \times 10^{3}$ cells/well and cultured for overnight. Then cells were treated with cisplatin as indicated concentrations. 24 or 48 hours later, $10 \mu \mathrm{L}$ CCK8 reagent (MedChemExpress ) was added into each well for another $2 \mathrm{~h}$. Then the absorbance was measured at 490nm using Cytation ${ }^{\mathrm{Tm}} 5$ Cell Imaging Multi-Mode Reader (Bio Tek).

\section{Flow cytometry}

For cell cycle analysis, cells were treated with cisplatin as indicated concentrations. After treated by cisplatin for $24 \mathrm{~h}$, cells were collected and fixed with $70 \%$ cold ethanol overnight. Then cells were washed with PBS and stained with $\mathrm{PI}$ at $37^{\circ} \mathrm{C}$ for 30 minutes. At last, the cell cycle was determined by using flow cytometry.

Annexin V-FITC cell apoptosis detection kit (Keygentec) was used to determine cisplatin-induced apoptosis in $\mathrm{H} 1299$ and $\mathrm{H} 460$ cells with altered SNRPB expression according to the manufacturer's instruction.

\section{Western blot}

Western blot assay was performed as previously described $[12,15]$. 


\section{Xenograft assay}

Six weeks old nude mice were subcutaneously injected with $\mathrm{H} 460$ cells $\left(5 \times 10^{6}\right.$ cells each mouse). Treatment was started when tumors reached $\sim 100 \mathrm{~mm}^{3}$ via tail vein injection of cisplatin $(5 \mathrm{mg} / \mathrm{kg})$. Tumor size was measured every 3 days. Thirty days after injection, mice were sacrificed and tumors were excised.

\section{Stastical analysis}

The results in this study were analized by GraphPad Prism 7.0 software. All data were shown as the means $\pm S D$. $P<0.05$ was considered statistically significant.

\section{Results}

\section{SNRPB regulates cisplatin-induced cell proliferation inhibition of NSCLC cells}

We first examine the SNRPB protein expression in a panel of 5 NSCLC cell lines. We find a higher expression level of SNRPB in $\mathrm{H} 1299$ and $\mathrm{H} 23$ cells and a lower expression level of SNRPB in H460 cells (Fig. 1A). We thus knock out of SNRPB in H1299 cells or overexpress it in H460 cells (Fig. 1B and 1C). Next, we test whether manipulate SNRPB expression can affect cisplatin-induced inhibition of cell growth in NSCLC cells using the CCK-8 assay. Consistent with our previous finding [12], overexpression of SNRPB promotes while knockout of SNRPB inhibits cell growth in non-treated H1299 and A549 cells, respectively (Fig. 1D, 1E). Interestingly, after the treatment with cisplatin for 24 or 48 hours, the inhibitory rate increased in H1299 cells with SNRPB knockout (Fig. 1D). Conversely, the inhibitory rate decreased in H460 cells with SNRPB overexpression (Fig. 1E). The cisplatin IC50 values were also determined in these two cell lines with or without SNRPB manipulation. As is shown in Fig. 1F, the IC50 increased from $16.56 \mu \mathrm{M}$ to $27.39 \mu \mathrm{M}$ when SNRPB knocked out in H1299 cells. However, the IC50 declined from $4.28 \mu \mathrm{M}$ to $2.46 \mu \mathrm{M}$ when SNRPB overexpressed in H460 cells (Fig. 1G). Taken together, these results indicate that SNRPB is a mediator of NSCLC cells in response to cisplatin.

\section{SNRPB regulates cisplatin response in NSCLC cells by affecting cell cycle progression}

To explore the potential mechanisms responsible for the function of SNRPB, cell cycle analyses were conducted by flow cytometry in $\mathrm{H} 1299$ and $\mathrm{H} 460$ cells. As is shown in Fig. 2A and 2B, cisplatin treatment increased the G1/G2 but decreased the S phase population of SNRPB knockout $\mathrm{H} 1299$ cells compared to control cells. However, overexpression of SNRPB decreased the percentage of G1/G2 but increased the percentage of $\mathrm{S}$ phase in $\mathrm{H} 460$ cells after treatment with cisplatin (Fig. $2 \mathrm{C}$ and 2D).

A previous study demonstrated that knockdown of SNRPB affects cancer genes expression, many of which are cell cycle-related genes [11]. We thus selected several dysregulated cell cycle-associated genes upon SNRPB knockdown for further confirmation and then examined whether their expression will 
change in SNRPB knocked out or overexpressed $\mathrm{H} 1299$ and $\mathrm{H} 460$ cells after treatment with cisplatin. Cisplatin treatment induced a much higher expression of CCNB1 and CDK4 in a dose-dependent manner in SNRPB knockout H1299 cells compared to control cells (Fig. 2E). However, overexpression of SNRPB reduced cisplatin-induced CCNB1 and CDK4 expression in $\mathrm{H} 460$ cells (Fig. 2F). These results suggest that SNRPB may modulate cell cycle progression of NSCLC cells by regulating cell cycle-related gene expression.

\section{Snrpb Modulates Cisplatin-induced Apoptosis In Nsclc Cells}

We next measured cisplatin-induced apoptosis by flow cytometry in $\mathrm{H} 1299$ and $\mathrm{H} 460$ cells with altered SNRPB expression. The results showed that knockout of SNRPB markedly decreased cisplatin-induced apoptosis in $\mathrm{H} 1299$ cells (Fig. 3A and 3B). While in H460 cells, overexpression of SNRPB significantly increased cisplatin-induced apoptosis compared to control cells (Fig. 3C and 3D). We also assessed the activity of the molecular marker of apoptosis PARP by western blotting assay. As shown in Fig. 3E, cisplatin treatment induced higher expression of cleaved PARP in SNRPB knockout $\mathrm{H} 1299$ cells compared to the wild type cells. However, ectopic expression of SNRPB in H460 cells decreased cisplatininduced cleaved PARP expression (Fig. 3F). We obtained similar results when examined the expression of the DNA damage marker $\mathrm{Y}-\mathrm{H} 2 \mathrm{AX}$ (Fig. 3E and 3F), suggesting that SNRPB negatively regulates cisplatininduced apoptosis in NSCLC cell.

\section{SNRPB regulates ERK signaling pathway activity in NSCLC cells after treatment with cisplatin}

Previous studies have reported that activated ERK signaling pathway is responsible for the resistance of NSCLC cells to cisplatin $[16,17]$. To investigate whether SNRPB regulates cisplatin resistance in NSCLC via ERK signaling pathway, we detected the pERK level in H1299 and A549 cells treated with multiple concentrations of cisplatin. As shown in Fig. 4A and 4B, cisplatin treatment activates ERK in a dosedependent manner in control H1299 cells. However, knockout of SNRPB significantly decreased the cisplatin-induced pERK level. In control of $\mathrm{H} 460$ cells, cisplatin treatment did not affect ERK pathway activity. While in SNRPB overexpressing $\mathrm{H} 460$ cells, high concentration $(0.5,1 \mu \mathrm{g} / \mathrm{ml})$ cisplatin could induce pERK expression (Fig. 4C, 4D). These results suggest that SNRPB may regulate the toxicity effect of cisplatin via ERK signaling pathway in the NSCLC cell.

\section{SNRPB-mediated xenograft NSCLC tumors were more effectively inhibited by cisplatin}

Due to SNRPB-knocked out H1299 cells could hardly form tumors in nude mice [12], we then tested whether SNRPB influence anti-tumor effects of cisplatin in H460-mediated xenograft tumor model. In this regard, control and SNRPB overexpressing $\mathrm{H} 460$ cells were subcutaneous injected into 6-week old nude mice. Tumor volume was measured 12 days after cancer cells injected. Consistent with our previous findings, overexpression of SNRPB significantly promote tumor growth in nude mice (H460-Vector Ns Vs H460-SNRPB Ns, Fig. 5A) [12]. Cisplatin treatment markedly reduced tumor growth in the SNRPBoverexpressing group. While in the vector expressing group, cisplatin treatment only had a modest impact 
on tumor growth (Fig. 5A). The tumor inhibition rate of cisplatin is higher in the SNRPB-overexpressing group than in the control group (H460-Vector + Cisplatin Vs H460-SNRPB + Cisplatin, Fig. 5B). Also, the tumor volume and weight are bigger and heavier in the SNRPB-overexpressing group than in the vectoroverexpressing group. However, cisplatin only has a significant impact on tumor growth when SNRPB overexpressed in tumor cells (Fig. 5C, 5D). These results suggest that SNRPB enhances cisplatin-induced tumor inhibition in vivo.

\section{Discussion}

SNRPB is a member of the Sm complex which consists of several other Sm proteins, SNRPD1, D2, D3, E, F, G [18]. Recent studies have been reported that many Sm proteins are involved in cancer progression. Targeting these proteins may trigger anti-tumor effects in man types of cancer. For instance, depletion of SNRPD and SNRPE inhibits cell viability via inducing autophagy in breast, lung and melanoma cancer cells [19]. Another study indicates that Small Nuclear Ribonucleoprotein Polypeptide A (SNRPA) contributes to gastric cancer progression via regulating NGF expression [20]. However, little is known about whether $\mathrm{Sm}$ proteins are involved in drug resistance in cancer.

Our previous studies indicated that SNRPB is highly expressed in NSCLC tissues compared to normal tissues. SNRPB promotes NSCLC cell proliferation and migration in vitro as well as xenograft tumor growth and metastasis in vivo. The knockout of SNRPB significantly attenuates cell proliferation and migration ability [12]. We, therefore, propose that knocking out of SNRPB may also sensitize NSCLC cells to chemotherapy agents, such as cisplatin. But to our surprise, we found that SNRPB negatively regulates cisplatin sensitivity in NSCLC cells. In the H1299 cell line with the high basal expression level of SNRPB, knocking out SNRPB could significantly decrease cisplatin-induced cell proliferation inhibition, cell cycle arrest and apoptosis. However, in the H460 cell line with the low basal expression level of SNRPB, overexpressing of SNRPB markedly increased cisplatin-induced cell proliferation inhibition, cell cycle arrest and apoptosis.

The knockdown of SNRPB affected multiple AKT and MAPK/ERK pathways gene expression in glioblastoma [11]. We then examined whether these two important oncogenic pathways were affected in NSCLC cells upon cisplatin treatment. Our results showed that SNRPB regulated ERK either than the AKT pathway (data not shown) activity in NSCLC cells under cisplatin treatment. The ERK signaling pathway plays an essential role in controlling the cell cycle by regulating downstream gene expression [21, 22]. It may be involved in SNRPB-mediated cell cycle-related gene expression in NSCLC cells upon cisplatin treatment. So our results suggest that SNRPB might modulate cisplatin sensitivity via regulation of ERK signaling pathway activity. But how SNRPB differently regulates ERK activity in NSCLC cells needs further study.

Cisplatin is one of the most important first-line chemotherapeutic drugs for NSCLC patients in the clinic $[23,24]$. However, some patients with NSCLC suffer severe adverse drug reactions with minimal effect [25]. So identifying molecular markers to predict the response of patients to cisplatin becomes important 
and necessary to improve the efficiency of cisplatin. Recent studies have identified several prediction markers for patients in response to cisplatin treatment, such as RASL11B for bladder cancer [26], ERCC1 for locally advanced cervical squamous cell carcinoma and ARID3B for head and neck squamous cell carcinoma $[27,28]$. In conclusion, our results may suggest a novel prediction marker for NSCLC patients in response to cisplatin-based chemotherapy.

\section{Abbreviations}

SNRPB

Small nuclear ribonucleoprotein polypeptides B And B'

NSCLC

Non-small-cell lung cancer

CCK-8

Cell counting kit-8

SCLC

Small cell lung cancer

CDDP

Cisplatin

DMEM

Dulbecco's Modified Eagle's Medium

FBS

Fetal bovine serum

IC50

The half maximal inhibitory concentration

ERK

Extracellular signal-regulated kinases

\section{Declarations}

\section{Ethics approval and consent to participate}

All the procedures of animal experiments were approved by the Animal Care Committee of Xuzhou Medical University.

\section{Consent for publication}

Not applicable.

\section{Availability of data and materials}

All data are included in the article. 


\section{Competing interests}

The authors report no conflicts of interest in this work.

\section{Funding}

This work was sppourted by grants from the National Natural Science Foundation of China (No. 81972165, No. 81972845).

\section{Authors' contributions}

NLL and LZZ designed this project and supervised the experiments. AXC, NF and XCL performed the experiments. NLLand AXC wrote the manuscript. All authors read and approved the final manuscript.

\section{Acknowledgements}

We thank Dr. Hui Qiu for hepful advices.

\section{References}

1. Mao Y, Yang D, He J, Krasna MJ. Epidemiology of Lung Cancer. Surg Oncol Clin N Am. 2016;25:43945.

2. Hashim D, Boffetta P, La Vecchia C, et al. The global decrease in cancer mortality: trends and disparities. Ann Oncol. 2016;27:926-33.

3. Gridelli C, Rossi A, Carbone DP, et al. Non-small-cell lung cancer. Nat Rev Dis Primers. 2015;1:15009.

4. Vansteenkiste J, Crino L, Dooms C, et al. 2nd ESMO Consensus Conference on Lung Cancer: earlystage non-small-cell lung cancer consensus on diagnosis, treatment and follow-up. Ann Oncol 2014; 25: $1462-1474$.

5. Schaake-Koning C, van den Bogaert W, Dalesio O, et al. Effects of concomitant cisplatin and radiotherapy on inoperable non-small-cell lung cancer. N Engl J Med. 1992;326:524-30.

6. Zaric B, Stojsic V, Tepavac A, et al. Adjuvant chemotherapy and radiotherapy in the treatment of nonsmall cell lung cancer (NSCLC). J Thorac Dis. 2013;5(Suppl 4):371-7.

7. Saltzman AL, Pan Q, Blencowe BJ. Regulation of alternative splicing by the core spliceosomal machinery. Genes Dev. 2011;25:373-84.

8. Wang BD, Ceniccola K, Hwang $\mathrm{S}$, et al. Alternative splicing promotes tumour aggressiveness and drug resistance in African American prostate cancer. Nat Commun. 2017;8:15921.

9. Wang Y, Bernhardy AJ, Cruz C, et al. The BRCA1-Delta11q Alternative Splice Isoform Bypasses Germline Mutations and Promotes Therapeutic Resistance to PARP Inhibition and Cisplatin. Cancer Res. 2016;76:2778-90.

10. Odunsi K, Mhawech-Fauceglia P, Andrews C, et al. Elevated expression of the serine-arginine protein kinase 1 gene in ovarian cancer and its role in Cisplatin cytotoxicity in vitro. PLoS One. 
2012;7:e51030.

11. Correa BR, de Araujo PR, Qiao M, et al. Functional genomics analyses of RNA-binding proteins reveal the splicing regulator SNRPB as an oncogenic candidate in glioblastoma. Genome Biol. 2016;17:125.

12. Liu N, Wu Z, Chen A, et al. SNRPB promotes the tumorigenic potential of NSCLC in part by regulating RAB26. Cell Death Dis. 2019;10:667.

13. Peng $\mathrm{N}, \mathrm{Li} \mathrm{J}, \mathrm{He} \mathrm{J}$, et al. c-Myc-mediated SNRPB upregulation functions as an oncogene in hepatocellular carcinoma. Cell Biol Int. 2020;44:1103-11.

14. Zhu L, Zhang $X$, Sun Z. SNRPB promotes cervical cancer progression through repressing p53 expression. Biomed Pharmacother. 2020;125:109948.

15. Liu N, Wu Z, Chen A, et al. ISG12a and its interaction partner NR4A1 are involved in TRAIL-induced apoptosis in hepatoma cells. J Cell Mol Med. 2019;23:3520-9.

16. Liu ZL, Jin BJ, Cheng CG, et al. Apatinib resensitizes cisplatin-resistant non-small cell lung carcinoma A549 cell through reversing multidrug resistance and suppressing ERK signaling pathway. Eur Rev Med Pharmacol Sci. 2017;21:5370-7.

17. Wu DW, Wu TC, Wu JY, et al. Phosphorylation of paxillin confers cisplatin resistance in non-small cell lung cancer via activating ERK-mediated Bcl-2 expression. Oncogene. 2014;33:4385-95.

18. Matera AG, Wang Z. A day in the life of the spliceosome. Nat Rev Mol Cell Biol. 2014;15:108-21.

19. Quidville V, Alsafadi S, Goubar A, et al. Targeting the deregulated spliceosome core machinery in cancer cells triggers mTOR blockade and autophagy. Cancer Res. 2013;73:2247-58.

20. Dou N, Yang D, Yu S, et al. SNRPA enhances tumour cell growth in gastric cancer through modulating NGF expression. Cell Prolif. 2018;51:e12484.

21. Chambard JC, Lefloch R, Pouyssegur J, Lenormand P. ERK implication in cell cycle regulation. Biochim Biophys Acta. 2007;1773:1299-310.

22. Yamamoto T, Ebisuya $M$, Ashida $F$, et al. Continuous ERK activation downregulates antiproliferative genes throughout G1 phase to allow cell-cycle progression. Curr Biol. 2006;16:1171-82.

23. Zhao Y, Zheng R, Chen J, Ning D. CircRNA CDR1as/miR-641/HOXA9 pathway regulated stemness contributes to cisplatin resistance in non-small cell lung cancer (NSCLC). Cancer Cell Int. 2020;20:289.

24. Fennell DA, Summers $Y$, Cadranel J, et al. Cisplatin in the modern era: The backbone of first-line chemotherapy for non-small cell lung cancer. Cancer Treat Rev. 2016;44:42-50.

25. Chan BA, Hughes BG. Targeted therapy for non-small cell lung cancer: current standards and the promise of the future. Transl Lung Cancer Res. 2015;4:36-54.

26. Takata R, Katagiri T, Kanehira M, et al. Predicting response to methotrexate, vinblastine, doxorubicin, and cisplatin neoadjuvant chemotherapy for bladder cancers through genome-wide gene expression profiling. Clin Cancer Res. 2005;11:2625-36.

27. Bai ZL, Wang YY, Zhe H, et al. ERCC1 mRNA levels can predict the response to cisplatin-based concurrent chemoradiotherapy of locally advanced cervical squamous cell carcinoma. Radiat Oncol. 
2012;7:221.

28. Zhong Q, Fang J, Huang Z, et al. A response prediction model for taxane, cisplatin, and 5-fluorouracil chemotherapy in hypopharyngeal carcinoma. Sci Rep. 2018;8:12675.

\section{Figures}

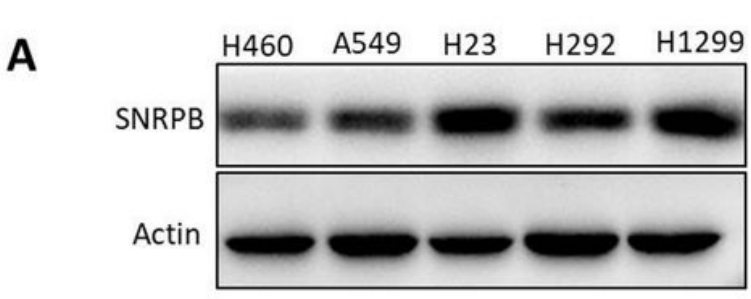

D
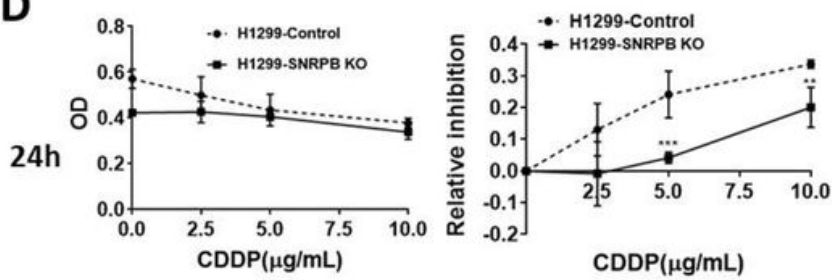

$48 \mathrm{~h}$
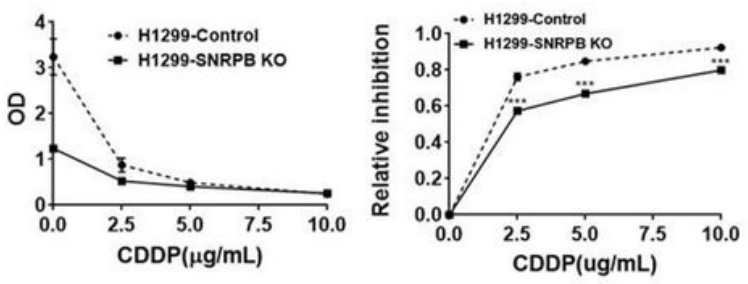

$\mathbf{F}$

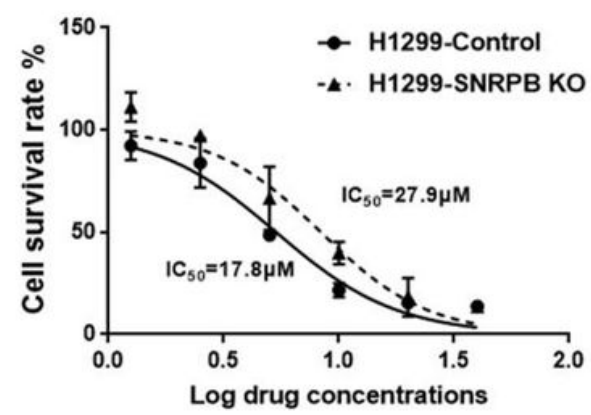

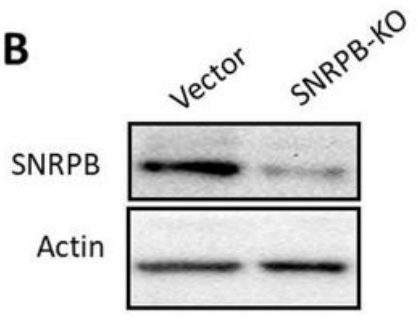

C

E
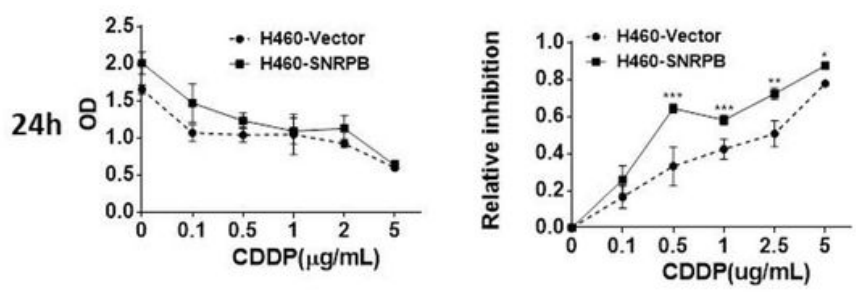

$48 \mathrm{~h}$
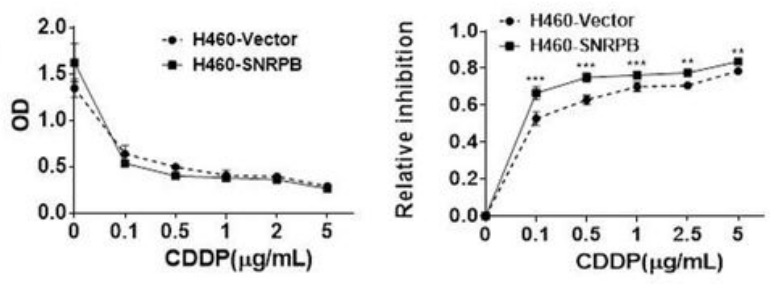

G

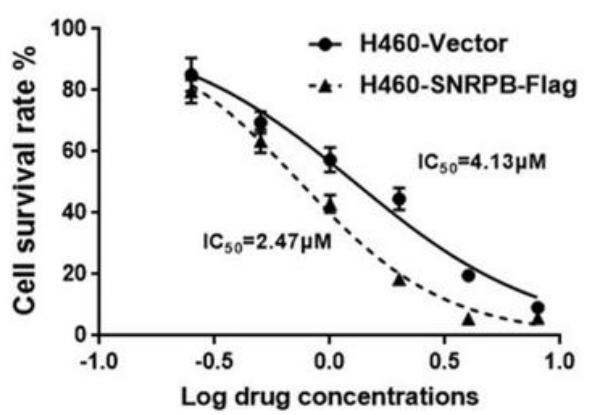

\section{Figure 1}

SNRPB regulates cisplatin-induced cell proliferation inhibition. (A) SNRPB expression was determined in six NSCLC cell lines via western blotting assay. (B) SNRPB knocked out in H1299 cells was confirmed via western blotting assay. (C) SNRPB overexpression in $\mathrm{H} 460$ cells was confirmed via western blotting assay. (D) H1299 control and SNRPB-KO cells were treated with cisplatin as indicated concentrations for 24 or 48 hours, cell proliferation was assessed via CCK8 assay by measuring the OD value (left panel). OD values were converted to relative inhibition values (right panel). (E) Similar assays were performed in 
H460-vector and H460-SNRPB cells. (F, G) The IC50 value of cisplatin was determined in control or SNRPB knocked out H1299 cells as well as in H460-vector and H460-SNRPB cells.

A

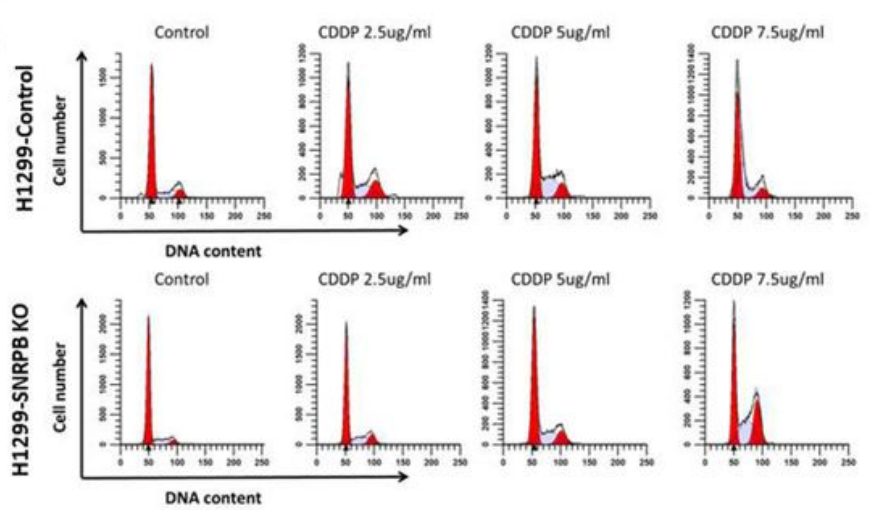

B

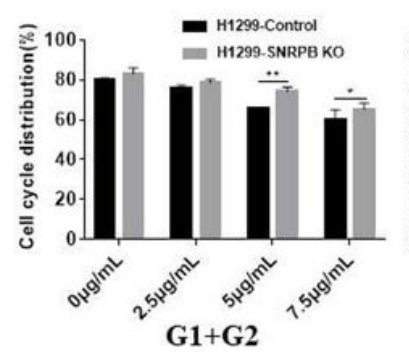

$\mathbf{E}$

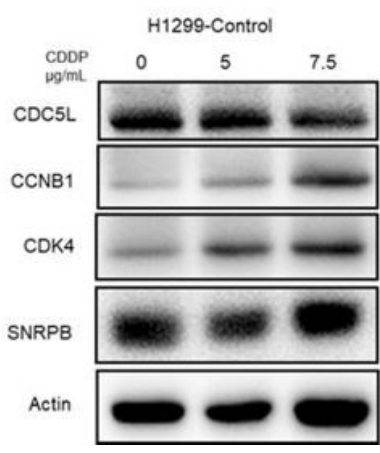

C
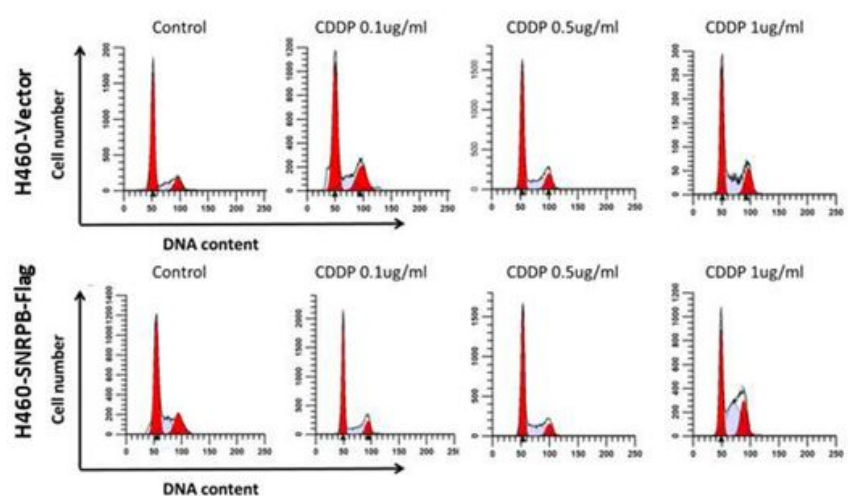

D
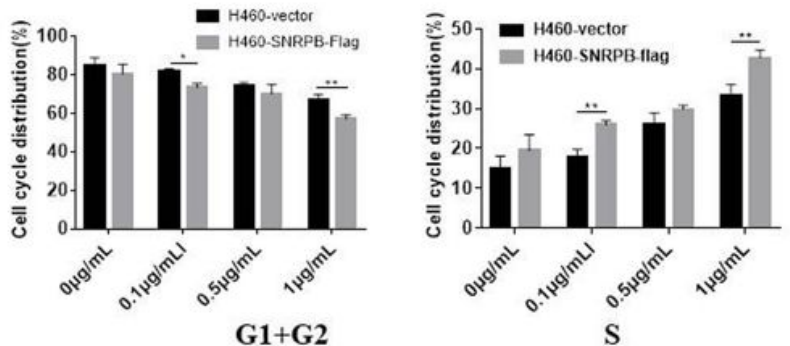

$\mathbf{F}$

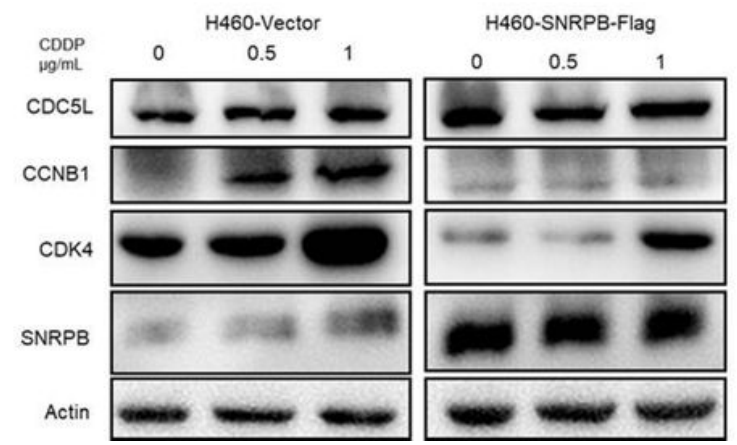

Figure 2

SNRPB regulates cisplatin-induced cell cycle arrest in NSCLC cells. (A) Cell cycle assays were performed via flow cytometry in control or SNRPB-knocked out H1299 cells treated with indicated concentrations of cisplatin for 24h. (B) Quantification of G1/G2 and S phase cells from A. (C) Similar experiments of A were performed in H460-vector and H460-SNRPB cells. (D) Quantification of G1/G2 and S phase cells from C. (E, F) Cell cycle-related proteins were examined by western blotting in $\mathrm{H} 1299$ and $\mathrm{H} 460$ cells treated with cisplatin for $24 \mathrm{~h}$. 


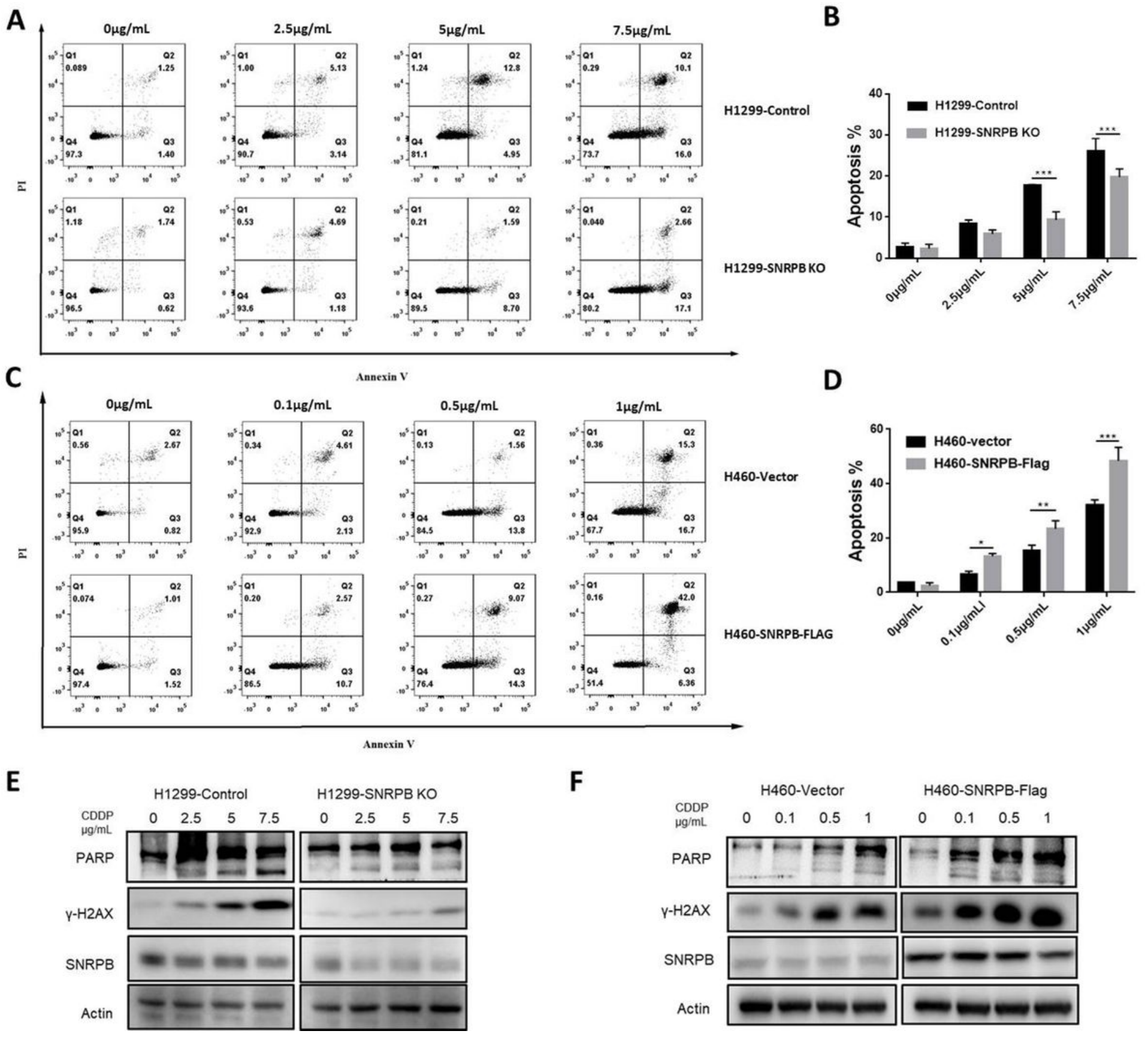

Figure 3

SNRPB regulates cisplatin-induced apoptosis in NSCLC cells. (A) Control and SNRPB-knocked out H1299 cells were treated with cisplatin as indicated concentrations for $24 \mathrm{~h}$, cell apoptosis was determined by flow cytometry. (B) Quantification of apoptosis in A. (C) Similar assays were performed as in A. (D) Quantification of apoptosis in C. (E, F) H1299 and H460 cells with altered SNRPB expression were treated with cisplatin as indicated concentrations for 24h. PARP, $\mathrm{Y}-\mathrm{H} 2 \mathrm{AX}$ and SNRPB expression were examined by western blotting. Actin used as a loading control. 
A

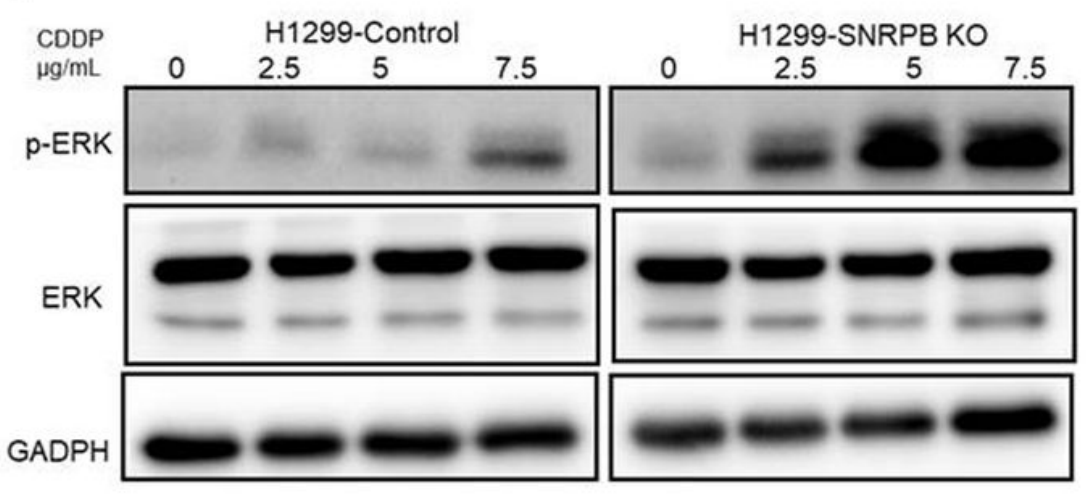

C

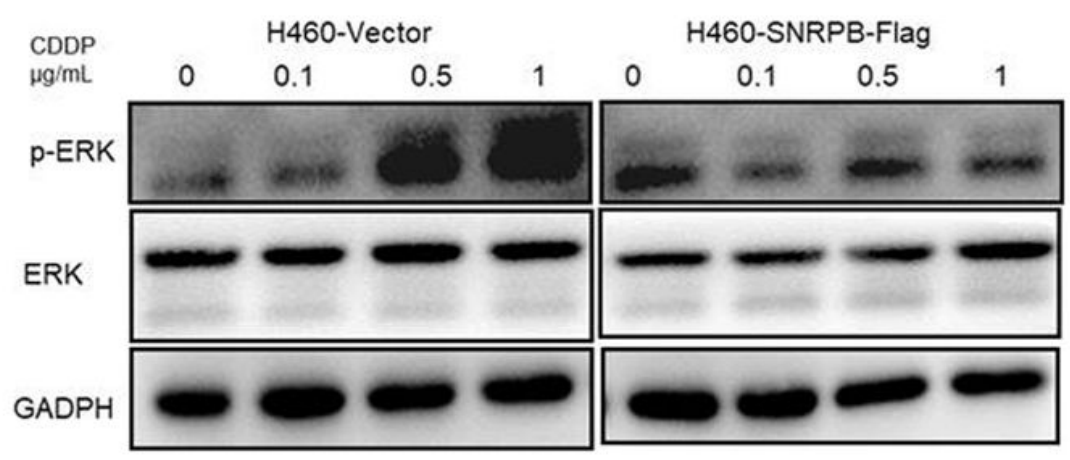

B
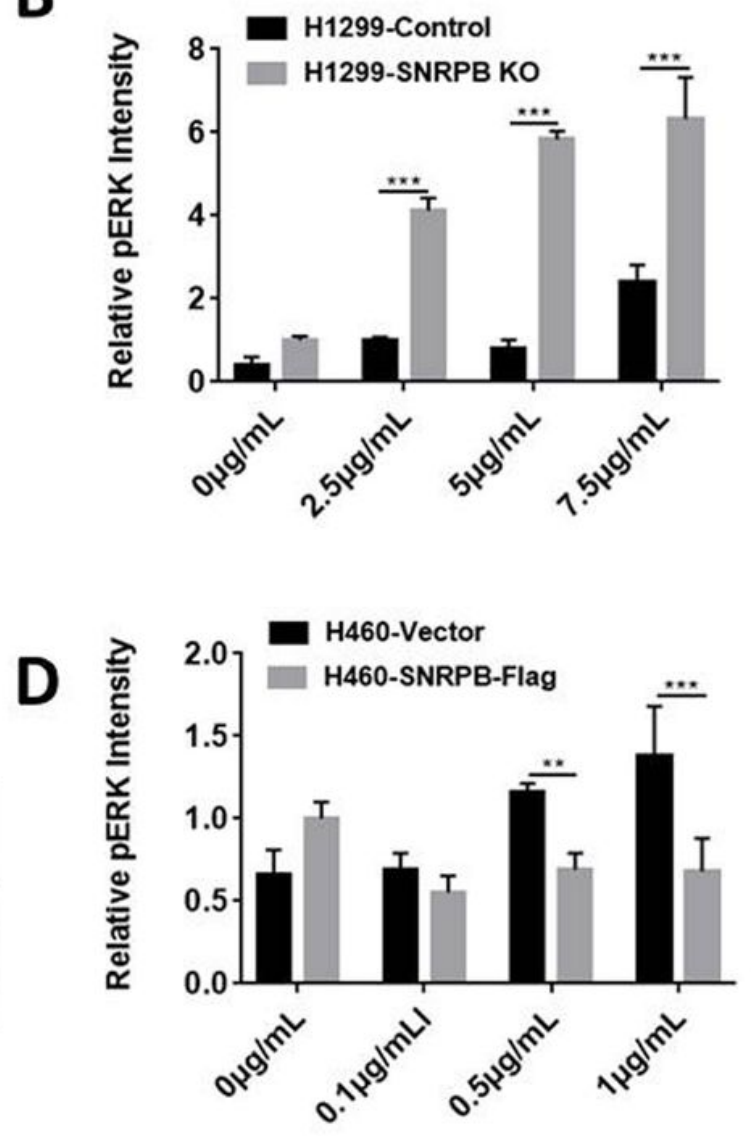

Figure 4

SNRPB regulates ERK activity in NSCLC cells under cisplatin treatment. (A) Control and SNRPB-knocked out $\mathrm{H} 1299$ cells were treated with cisplatin as indicated concentrations for 24h, pERK and ERK expression were assessed by western blotting. GAPDH was used as a loading control. (B) Quantification of pERK expression in A. (C) H460-vector and H460-SNRPB cells were treated with cisplatin as indicated concentrations for $24 \mathrm{~h}$, pERK and ERK expression were assessed by western blotting. GAPDH was used as a loading control. (D) Quantification of pERK expression in C. 
A

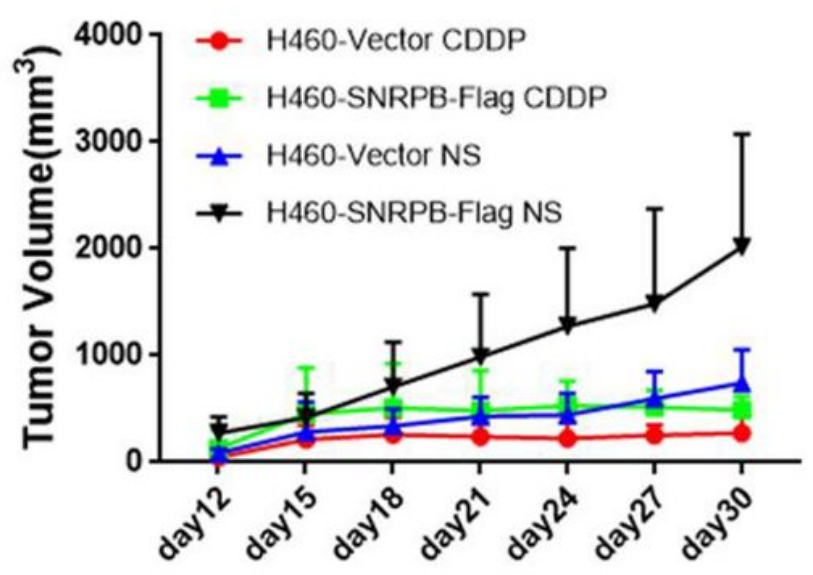

C

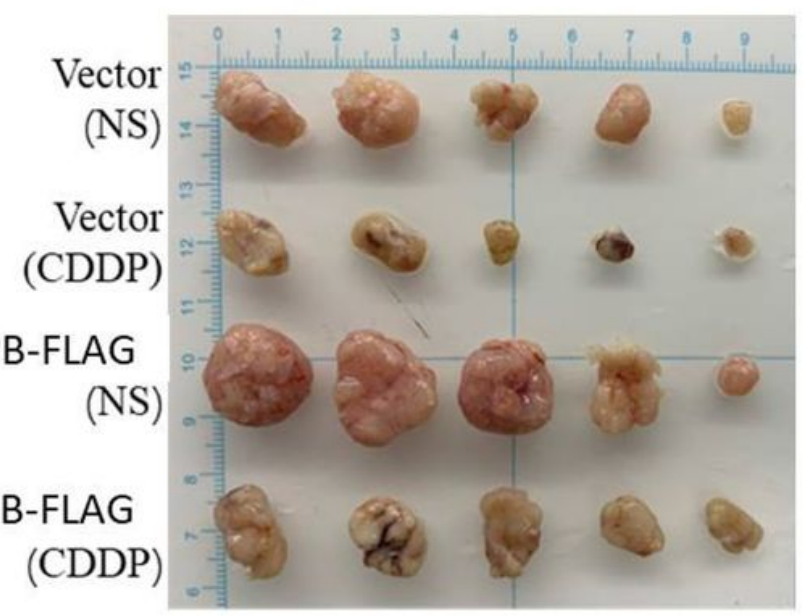

B

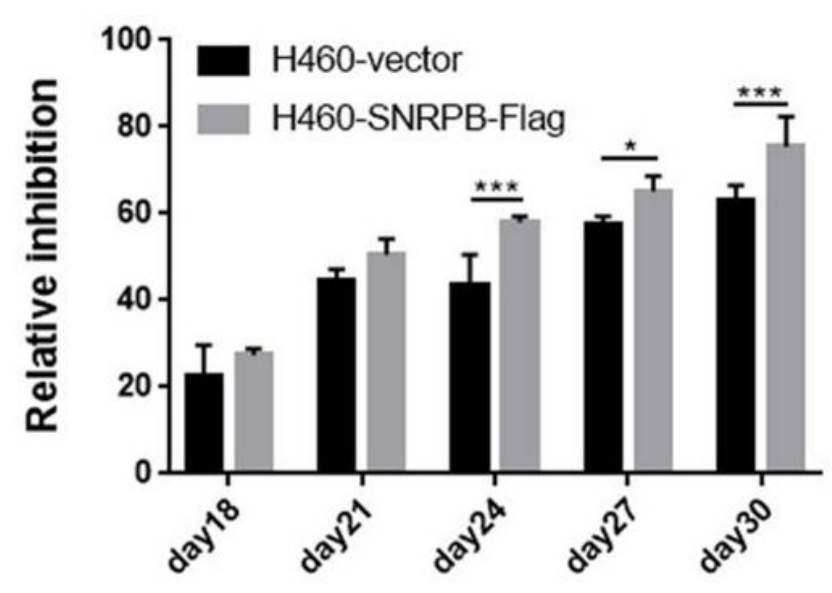

D

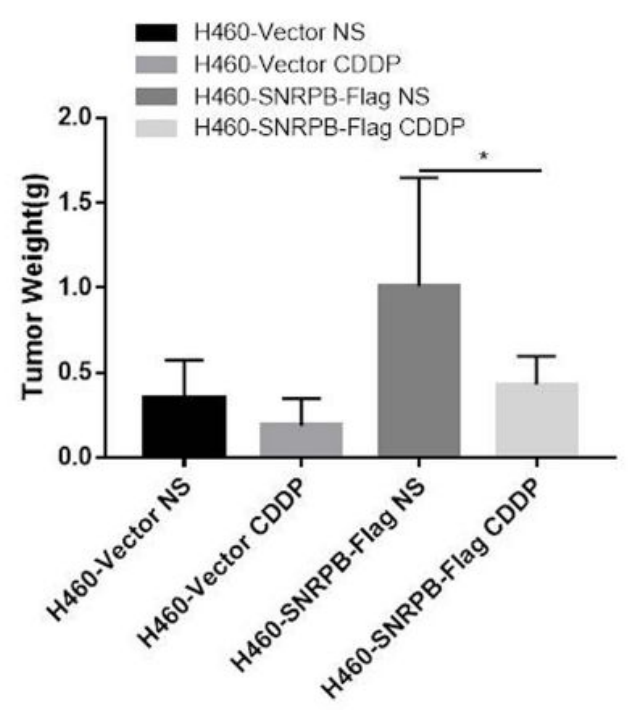

Figure 5

SNRPB overexpression enhances cisplatin-induced tumor inhibition. (A) Six weeks old nude mice were injected with $5 \times 106 \mathrm{H} 460$ cells. Cisplatin $(5 \mathrm{mg} / \mathrm{kg}$ ) was intraperitoneally injected when tumors reached $\sim 100 \mathrm{~mm} 3$. Tumor sizes were measured every 3 days. (B) Relative inhibition values at days 18, 21, 24, 27 and 30 converted from (A) were shown. (C) Tumors of each group were shown after mice sacrificed on day 30. (D) The tumor weight of each group was measured after mice sacrificed on day 30. 